\title{
AN APPROACH FOR CHOOSING THE COST EFFECTIVE DESIGN FOR A PRODUCT-SERVICE SYSTEM WHILE MAINTAINING ITS DESIRED RELIABILITY
}

\author{
Schneider, Jannik Alexander; Mozgova, Iryna; Lachmayer, Roland \\ Leibniz University Hannover
}

\begin{abstract}
With the spread of product-service systems as business models the life cycle costs are of increasing importance as a measurement of product cost. A key factor that drives these costs is the desired reliability of the products used to provide the service. Since the customer usually expects as uninterrupted service availability, it is imperative to achieve the the required reliability. Therefore a large variety of methods has been developed to maximize the reliability of a product. But these approaches focus on the maximization of the reliability and disregard the resulting product costs. This can lead to designs that over perform concerning their reliability requirements but also exceed their target costs. Which will result in the product-service system not being competitive in the marketplace or lowering the company's profit. This paper shows an approach on how to use markov chains to enable a quick comparison of life cycle costs from different product-service system designs With this it will be possible to make better informed decisions about the costs of a system while still meeting the reliability targets.
\end{abstract}

Keywords: Product-Service Systems (PSS), Evaluation, Design costing, Life cycle costing, Reliability

Contact:

Schneider, Jannik Alexander

Leibniz University Hannover

Institut für Produktentwicklung und Gerätebau

Germany

schneider@ipeg.uni-hannover.de

Cite this article: Schneider, J.A., Mozgova, I., Lachmayer, R. (2019) 'An Approach for Choosing the Cost Effective Design for a Product-Service System While Maintaining its Desired Reliability', in Proceedings of the 22nd International Conference on Engineering Design (ICED19), Delft, The Netherlands, 5-8 August 2019. DOI:10.1017/dsi.2019.311 


\section{INTRODUCTION}

A major factor for many key decisions of market driven companies is the profit that can be expected by those decisions. Since profit is determined by costs and revenue the objective is to achieve the maximum revenue with the minimal amount of costs necessary. Common ways to reduce the cost of product are for example developing cost-efficient products or rationalizing the manufacturing process (Ehrlenspiel et al., 2007). The increase of revenue is impacted by product/service quality, number of sales or improvements in delivery time. To achieve the maximal possible profit revenue as well as cost factors need to be considered and trade-offs weight. As a relatively new concept the product service system has been proposed by Goedkoop. In this case the customer is not solely sold a product but a combination of a product and a service (Goedkoop, 1999). With the further development of this concept approaches have been developed where the actual service is more in the focus of the customer than the product providing it. For those product service systems with a high service component Tukker has shown that it is vital to take the life cycle cost into consideration, since the products and their costs remain with the manufacturer (Tukker, 2004). These life cycle costs are defined as "discounted cumulative total costs incurred by a specified function or item of equipment over its life cycle" (ISO 15663-1, 2000). Which results in a necessity to view costs over the entire lifespan of a product, the accumulating operational costs of a product are most often larger than the one-time capital costs (Farr, 2011). Therefore, when developing a product for use in product-service systems, in addition to the one time capital costs of a product, the costs generated during the operation of product are of high significance (Johannknecht et al., 2016a). Apart from the cost of a product the customers' willingness to purchase said product are directly tied to the characteristic of the product. A key customer expectation is that the delivery of the purchased service to be uninterrupted (Johannknecht et al., 2016b). The determining factors for enabling this is the reliability of the product used to deliver the service (Eberlin and Hock, 2014). Consequently reliability is an important factor to be considered during product development. In the case of a single product unit the only option is to increase the reliability of the product to achieve the required reliability. In the case of the product service system there is another option instead of increasing the reliability of a product one can supply an increased number of identical products as cold redundancy, meaning having additional instances of the product available a replacement and those experiencing no load, to achieve the desired reliability for the service.

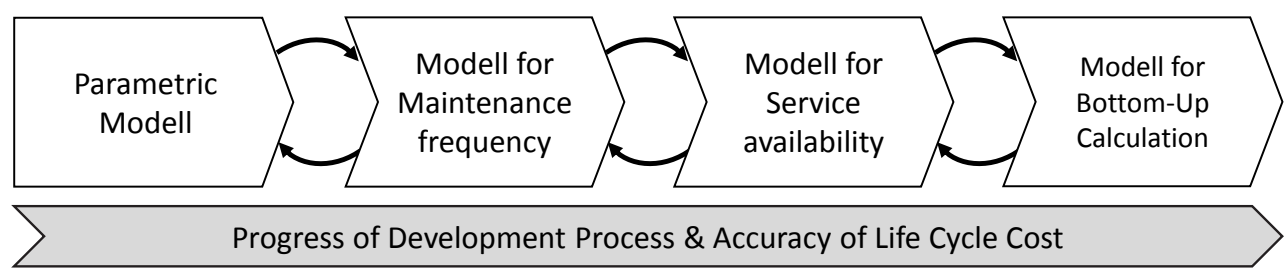

Figure 1. Modell for cost prognostication (Johannknecht, 2018)

The necessity to consider the availability of service for cost prognostication of product-service systems is shown in Figure 1 by Johannknecht. This is necessary because on the one hand it impacts customer satisfaction but furthermore creates indirect opportunity costs. The approach shown in (Johannknecht, 2018) focuses on how differently designed products can impact the availability of service. But it does not consider the option of using multiple products as redundancies to ensure service availability. This paper gives a method on how to compare the costs between those two different approaches for achieving service availability.

\section{STATE OF THE ART}

Methods to calculate the reliability of a product or system are numerous. These methods are described in many different technical standard literature like (Eberlin and Hock, 2014) and (Ebeling, 2010). The aforementioned methods are designed to calculate system reliability using given values for their subcomponents. In this case it is important to determine whether the components are connected in series, parallel or some combination of both. Recent research has also been exploring the possibility of optimizing system reliability and cost (Coit, 2003; Chambari et al., 2012). Due to the fact that this 
problem is part of the class of NP-hard optimization problems (Chern, 1992) metaheuristic algorithms have been used to find solutions. Depending on the application scenario this can result in a large number of constraints that have to be modelled which are based on the real design constraints. A more general approach is described by Ehrlenspiel et al. (2007) and broken down into three main steps; the clarification of task, the discovery of possible solutions and the selection of a solution. The step of selecting a solution is what needs to be considered here since the questions is whether increased costs for improved reliability are economically viable or a cold standby solution is to be preferred. A proven method for evaluating the reliability of standby solutions is the usage of Markov chains to determine the probabilities of service being available or unavailable (Ebeling, 2010). Individual machine reliability is not relevant in this case since the customer of a product service system only perceives the reliability of the service provided for him and not the reliability of the machine providing said service. These existing approaches show the cost of individual products with respect to their reliability. In the case of product-service systems, it is possible to use more than one product to achieve a desired availability of a service sold to customers. The approach shown in chapter 3 demonstrates how to evaluate quickly whether two cheaper and individually less reliable products can be a better solution than a more expensive product with increased reliability. Assuming the availability requirement and all other fixed requirements will be full filled by both options, the profitability is the key determining factor for deciding which design concept is preferable.

\subsection{Markov analysis}

Markov chains are used to describe the different states of a system and how the system transfers in between those different states. It is assumed that the change between any two given states is instant and requires no time it self (Stewart, 2009; Norris, 1997). Markov chains can be divided into two distinct groups; the Markov process for cases with a continuous state-space and the Markov chain for case with a discrete state-space (Ross, 2014; Stewart, 2009). The Markov chain is a suitable solution for the proposed approach the cases in which the service is provided have to be compared with those in which the service is unavailable.

A frequently used example for markov chains is the weather. In this case three different states are defined; $\mathrm{R}$ for rainy, $\mathrm{C}$ for cloudy and $\mathrm{S}$ for sunny. For each of these states a probability exist to change into a different state or to remaining in the same state, these transitional probabilities are denoted as $P_{i j}$; e.g. $p_{R S}=0.2$.

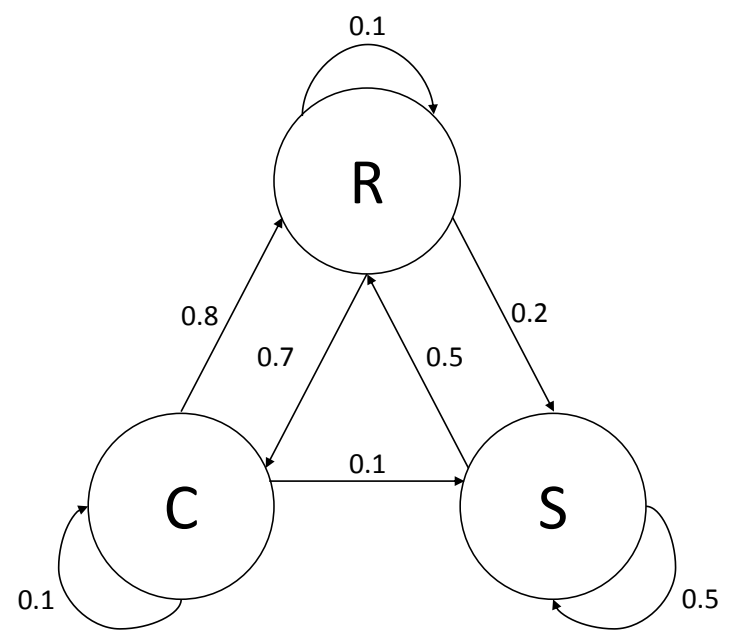

Figure 2. Markov chain for weather (Stewart, 2009)

The probabilities shown in Figure 2 can be noted as a matrix called the Transition matrix $P$. For this the states have to be assigned to the rows and columns of the matrix. In this case $\mathrm{R}, \mathrm{C}$ and $\mathrm{S}$ were chosen as columns from left to right and rows from top to bottom, resulting in the matrix shown in Equation (1). This displays all transition probabilities as a change between the state of the row to state of the column: 


$$
P=\left(\begin{array}{ccc}
0.1 & 0.7 & 0.2 \\
0.8 & 0.1 & 0.1 \\
0.5 & 0 & 0.5
\end{array}\right)=\left(\begin{array}{lll}
p_{R R} & p_{R C} & p_{R S} \\
p_{C R} & p_{C C} & p_{C S} \\
p_{S R} & p_{S C} & p_{S S}
\end{array}\right) .
$$

With a known start point given as a vector it is possible to calculate the probability for the system being in any of the other states after one unit of time has passed and one transition has happened. The system can be in one of three different states it is either rainy, cloudy or sunny these different system states can be denoted as a vector $v^{0}$ with the exponent denoting how many system changes have already happened at that point. Equation (2) shows that if a rainy day is the start point the probability for the next day either being rainy, cloudy or sunny are exactly equal to the probability of reaching any of those states from the rainy state:

$$
v^{0} P=v^{1}=\left(\begin{array}{lll}
0.1 & 0.7 & 0.2
\end{array}\right) \text { with } \quad v^{0}=\left(\begin{array}{lll}
1 & 0 & 0
\end{array}\right) .
$$

This way it is possible to calculate the probability for the system to be in any of its states after a given amount of time $t$. Equation (3) show that to determine the state of the system after a given amount of time all previous system states starting with the current have to be calculated:

$$
v^{t-1} P=v^{\mathrm{t}} \text {. }
$$

However, if a Markov chain is irreducible, this means that a stationary distribution is calculable. Given this information it can be shown that for large values of $t$ the vector $v^{t}$ converges onto a set of state probabilities that remain, even after an additional unit of time has passed. A Markov chain is irreducible if it is possible to reach any state from any other state.

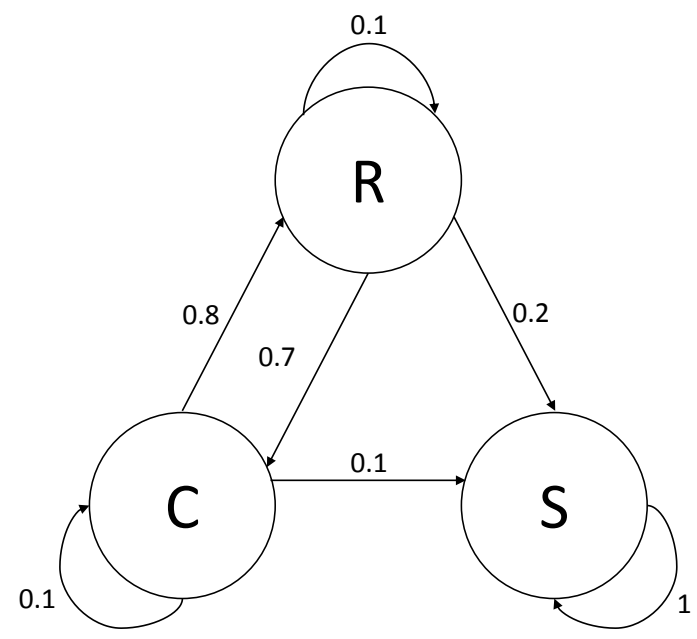

Figure 3. Modification of the embedded markov chain for weather.

The markov chain displayed in Figure 2. is irreducible, while the modification presented in Figure 3. is reducible. Once the system reaches the state sunny for the first time it will always remain in that state, because of the given possibility of 2. Therefore, for Figure 2 a stationary distribution can be calculated. For this, the identity matrix $I$ has to be subtracted from Equation (1). The outcome then is equal to zero since it is unclear in which state the system starts:

$$
v P-I=v\left(\begin{array}{ccc}
-0.9 & 0.7 & 0.2 \\
0.8 & -0.9 & 0.1 \\
0.5 & 0 & -0.5
\end{array}\right)=0 \text {. }
$$

One information that is missing from Equation (4) is that the sum of the probabilities for being in any given state is equal to 1 . To reflect this information an additional row is added and filled with ones this new matrix is called $Q$. Also the resulting vector is no longer equal to zero but to a vector $b=\left(\begin{array}{llll}0 & 0 & 0 & 1\end{array}\right)$, because the sum of the system being in any of its states is always equal to one. After taking into account this additional information the result is Equation (5): 


$$
v Q=v\left(\begin{array}{ccc}
-0.9 & 0.7 & 0.2 \\
0.8 & -0.9 & 0.1 \\
0.5 & 0 & -0.5 \\
1 & 1 & 1
\end{array}\right)=b
$$

Now Equation (5) needs to be solved for $v$. To achieve that both sides are multiplied with $Q^{\mathrm{T}}$ and then with $\left(Q Q^{T}\right)^{-1}$ which results in the Equation (6) and the stationary probabilities for each state:

$$
v=b Q^{T}\left(Q Q^{T}\right)^{-1}
$$

\section{APPROACH FOR A COST COMPARRISON OF RELIABILITY AND REDUNDANCY}

There are two major options for the service provider to achieve the required reliability for the service they provide. The first solution would be to improve the reliability of the individual machine used to deliver the service to a level that matches the requirements. Another solution is to use a standby machine that will take the place in case of failure of the first machine and ensures that the service can still be provided. This standby solution can be broken down into three different standby solutions cold redundancy, hot redundancy and warm redundancy. All of these redundancy types have a different reaction time after failure as well as a different load during standby. In all of these cases a further differentiation is necessary to decide whether a preference for either of the machines exists. In case of a preference the machines will be switched over immediately when the preferred machine becomes available again.

In order to effectively compare these different options, the reliability of each option needs to be converted into an economically comparable number, taking into account all cost components. First of all the costs for the individual machine $C_{m n}$ have to be considered. Additionally the cost for the machine in operation $C_{a n}$ have to be taken into account in the case that not all machines have the same costs of operation $n$ signifies the number of the machine for which these costs are relevant. With that all costs unaffected by reliability are considered in the comparison and only those that are affected by reliability remain. The cost of repair $C_{r n}$ is driven by the reliability of the machine because its reliability determines how often a repair has to be performed. In some cases switching from one machine to another one is because replacing the non-functioning machine with the functioning one is associated with the $\operatorname{cost} C_{s n}$.

Table 1. Notations for Equation (7)

\begin{tabular}{|l|l|l|l|l|l|l|}
\hline Notation & $C_{m n}$ & $C_{a n}$ & $C_{r n}$ & $C_{s n}$ & $C_{o n}$ & $C_{p}$ \\
\hline Cost for & a machine & operation & repair & switching machines & opportunity & production stop \\
\hline
\end{tabular}

An example for $C_{s n}$ would be removing an aircraft engine from a wing and replacing it will require work and therefore incur costs. Furthermore owning an asset and holding it in standby to use in case of a failure of the other machine means that the machine in standby is not creating revenue and therefore these costs should be considered opportunity costs $C_{o n}$. Finally it has to be considered that in case of a failure of a machine and an inability to continue the service a loss in revenue occurs and a financial penalty set in the contract with the customer may be enforced. This will be denoted as $C_{p}$. The combination of these costs is the total cost for service $C_{T}$. The cost will be multiplied with the probability of the system being in that given state. For example $P_{a n}$ as the probability of any given machine being active:

$$
C_{T}=C_{m n}+\sum_{n=1}^{N} C_{a n} * P_{a n}+\sum_{n=1}^{N} C_{r n} * P_{r n}+\sum_{n=1}^{N} C_{s n} * P_{s n}+\sum_{n=1}^{N} C_{o n} * P_{o n}+C_{p} * P_{p} .
$$

After inputting all the costs and probabilities in Equation (7) its result $C_{T}$ is easily comparable and allows for the selection of the option with the lowest cost. Since the equation is used to calculate costs, it is the result with the smallest value that should be preferred. 


\subsection{Single machine for service delivery}

In the case of a single machine there are only two relevant states that have to be defined, the machine is active and working displayed as state $A$ and the second state $r$ shows the machine as in maintenance and therefore unable to provide the service with the notation $r$. The $\lambda_{1}$ in Figure 4 stands for the average failure rate of machine one. While $\mu_{1}$ stands for the average repair rate for machine one. Opportunity cost are not relevant in this case and can be omitted from Equation (7). The switchover cost can be omitted as well since there are no machines to switch between.

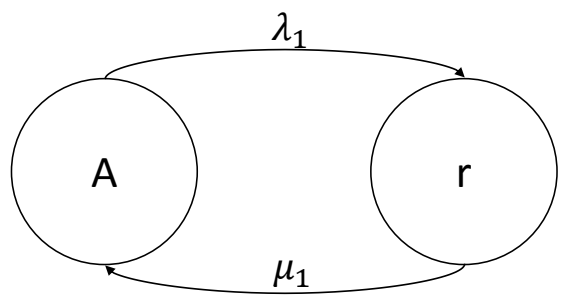

Figure 4. Single machine for service delivery

Three probabilities have to be determined; $P_{a n}, P_{m}$ and $P_{p}$, whereas Figure 4 shows only two distinct states the machine is in. This is because with one machine the state $r$ represents both the machine being in repair and the production not being possible. Therefore, in this case $P_{r n}=P_{p}$ which means all necessary probabilities can be determined. With the transition diagram shown in Figure 4 the following transition matrix $P$ is created:

$$
P=\left(\begin{array}{cc}
1-\lambda_{1} & \lambda_{1} \\
\mu_{1} & 1-\mu_{1}
\end{array}\right) .
$$

Multiplying this with the vector for the state probabilities and recalling that the system has to be in one of both states this information can be written as in Equations (9), (10) and (11):

$$
\begin{aligned}
& P_{a 1}=P_{a 1} *\left(1-\lambda_{1}\right)+P_{r 1} * \mu_{1}, \\
& P_{r 1}=P_{a 1} * \mu_{1}+P_{r 1} *\left(1-\mu_{1}\right), \\
& P_{a 1}+P_{r 1}=1 .
\end{aligned}
$$

With the given information in Equations (9), (10) and (11) the solution for $P_{a 1}$ and $P_{r 1}$ can be found as the following values:

$$
\begin{aligned}
& P_{a 1}=\frac{\mu_{1}}{\lambda_{1}+\mu_{1}}, \\
& P_{r 1}=\frac{\lambda_{1}}{\lambda_{1}+\mu_{1}} .
\end{aligned}
$$

Now all necessary informations are available and therefore can be inserted into Equation (7) as appropriate to calculate the cost of service for a single machine providing said service:

$$
C_{T}=C_{m 1}+C_{a 1} * \frac{\mu_{1}}{\lambda_{1}+\mu_{1}}+C_{r 1} * \frac{\lambda_{1}}{\lambda_{1}+\mu_{1}}+C_{p} * \frac{\lambda_{1}}{\lambda_{1}+\mu_{1}} .
$$

Looking at Equation (14) intuitive thoughts about the problem are confirmed. For an increase in machine reliability the value for $\lambda_{1}$ would move towards zero which results in the costs for repair and inability to provide the service lose in relevance or even get removed entirely for a machine with $100 \%$ reliability. Conversely for an increase in failure rate these costs would grow in relevance and the cost for an active machine would decrease since it spends less of its time active. Because of this it is technically possible that a machine that is never active incurs less cost than a machine that is active and therefore generating revenue. This can be avoided if $C_{p}$ is a value that is greater than $C_{a 1}$ then an 
active machine is always better than an inactive machine. If this system is to be compared to another one it is important to note that $C_{p}$ has to be identical between both options otherwise the results would be inaccurate.

\subsection{Two machines for service delivery}

Using a second machine in standby is another option to increase the reliability of the service and an option which could provide lower costs while still allowing for the required availability of the service. The machines used in this case can either be of identical specifications or be different. For example already phased out machines can be used as standby machines in order to minimize opportunity costs. Therefore this example will look at the option that both machines are different since this solves the problem for identical machines as well. Figure 5 shows all possible transitions between the different states, while not showing the probabilities for remaining in any given state. The state Aa is used to show the case that machine 1 is working while machine 2 is waiting in cold standby. Conversely state aA is denoting the opposite case that machine 2 is working and machine 1 is waiting in cold standby. Same holds true for the states $\mathrm{rA}$ and Ar where the position of $\mathrm{r}$ shows which machine is in repair and A which machine is working. Lastly rr represents the case that both machines are in repair and no work can be performed.

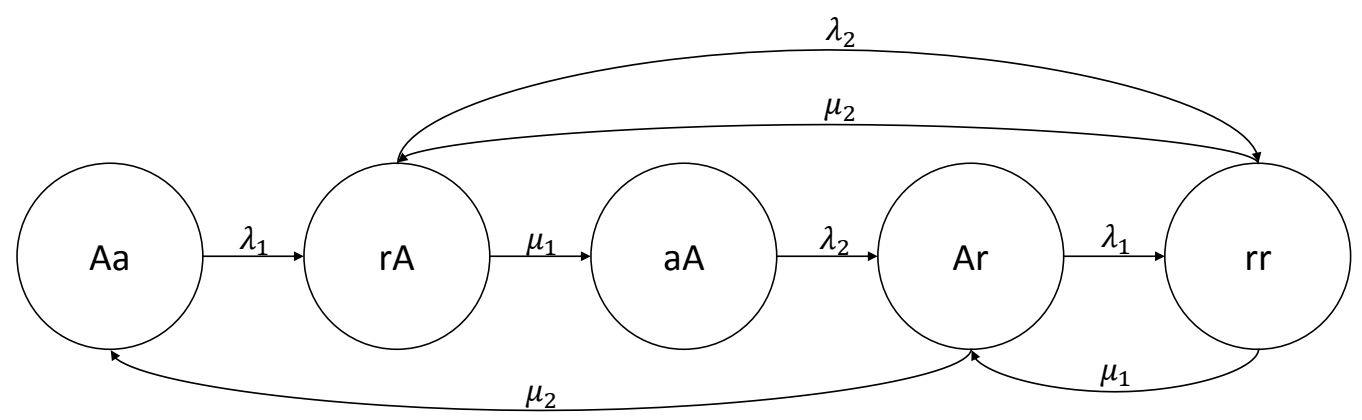

Figure 5. Two machines for service delivery with cold standby and no preference

These probabilities are calculated as the complementary probability of all probabilities leaving any given state. Inserting this information into matrix form the resulting matrix is shown in Equation (15):

$$
P=\left(\begin{array}{ccccc}
1-\lambda_{1} & \lambda_{1} & 0 & 0 & 0 \\
0 & 1-\lambda_{2}-\mu_{1} & \mu_{1} & 0 & \lambda_{2} \\
0 & 0 & 1-\lambda_{2} & \lambda_{2} & 0 \\
\mu_{2} & 0 & 0 & 1-\lambda_{1}-\mu_{2} & \lambda_{1} \\
0 & \mu_{2} & 0 & \mu_{1} & 1-\mu_{1}-\mu_{2}
\end{array}\right) .
$$

The Equation (15) can be solved using the methods described in section 2.1 of this paper. The resulting probabilities for the states will correspond to those shown in Figure 5:

$$
\begin{aligned}
& P_{A a}=\frac{\mu_{2}}{\lambda_{1}} * \frac{\mu_{1}\left(\lambda_{2}+\mu_{1}+\mu_{2}\right)}{\lambda_{1}\left(\lambda_{2}+\mu_{1}\right)+\lambda_{2} \mu_{2}} * \frac{\lambda_{1} \lambda_{2}}{\rho}, \\
& P_{r A}=\frac{\mu_{2}\left(\lambda_{2}+\mu_{1}+\mu_{2}\right)}{\lambda_{1}\left(\lambda_{2}+\mu_{1}\right)+\lambda_{2} \mu_{2}} * \frac{\lambda_{1} \lambda_{2}}{\rho}, \\
& P_{a A}=\frac{\mu_{1}}{\lambda_{2}} * \frac{\mu_{2}\left(\lambda_{2}+\mu_{1}+\mu_{2}\right)}{\lambda_{1}\left(\lambda_{2}+\mu_{1}\right)+\lambda_{2} \mu_{2}} * \frac{\lambda_{1} \lambda_{2}}{\rho}, \\
& P_{r A}=\frac{\mu_{1}\left(\lambda_{2}+\mu_{1}+\mu_{2}\right)}{\lambda_{1}\left(\lambda_{2}+\mu_{1}\right)+\lambda_{2} \mu_{2}} * \frac{\lambda_{1} \lambda_{2}}{\rho},
\end{aligned}
$$




$$
P_{r r}=\frac{\lambda_{1} \lambda_{2}}{\rho}
$$

with

$$
\begin{aligned}
\rho= & \left(\left(\lambda_{1} \lambda_{2}\left(\lambda_{2}+\mu_{1}\right)\left(\lambda_{2}+\mu_{1}+\mu_{2}\right)\right)+\left(\lambda_{1} \mu_{1} \mu_{2}\left(\lambda_{2}+\mu_{1}+\mu_{2}\right)\right)+\left(\lambda_{1} \lambda_{2} \mu_{2}\left(\lambda_{1}+\mu_{2}\right)\right)+\right. \\
& \left.+\mu_{1} \mu_{2}\left(\lambda_{1} \mu_{1}+\lambda_{2}\left(\lambda_{2}+\mu_{1}+\mu_{2}\right)\right)\right) / \lambda_{1}\left(\lambda_{2}+\mu_{1}\right)+\lambda_{2} \mu_{2} .
\end{aligned}
$$

These equations are used for any case where the failure and repair rates of the given machines are not equal. For the case that both machines have identical failure and repair rates the equations simplify to the following.

$$
\begin{aligned}
& P_{A a}=\frac{\mu^{2}}{2 \mu^{2}+2 \mu \lambda+\lambda^{2}}, \\
& P_{r A}=\frac{\lambda \mu}{2 \mu^{2}+2 \mu \lambda+\lambda^{2}}, \\
& P_{a A}=\frac{\mu^{2}}{2 \mu^{2}+2 \mu \lambda+\lambda^{2}}, \\
& P_{r A}=\frac{\lambda \mu}{2 \mu^{2}+2 \mu \lambda+\lambda^{2}}, \\
& P_{r r}=\frac{\lambda^{2}}{2 \mu^{2}+2 \mu \lambda+\lambda^{2}} .
\end{aligned}
$$

Now the probabilities that are found have to be matched with the notation in Equation (7). The case $P_{A a}$ has one machine active and machine two in standby and $P_{a A}$ with the machines switched the probabilities for the switch over costs are connected to the states $P_{r A}$ and $P_{A r}$. While $P_{r r}$ only sees the repair probabilities and the one for production loss.

Table 2. Connection between Figure 5. And Equation (7)

\begin{tabular}{|l|c|c|c|c|c|}
\hline$P_{i j}$ in Figure 4. & $P_{A a}$ & $P_{r A}$ & $P_{a A}$ & $P_{A r}$ & $P_{r r}$ \\
\hline $\begin{array}{l}\text { Corresponding } P_{i j} \text { in } \\
\text { Equation (1) }\end{array}$ & $P_{a 1} ; P_{o 2}$ & $P_{a 2} ; P_{r 1} ; P_{s 1}$ & $P_{a 2} ; P_{o 1}$ & $P_{a 1} ; P_{r 2} ; P_{s 2}$ & $P_{r 1} ; P_{r 2} ; P_{p}$ \\
\hline
\end{tabular}

With the information from Table 2 it is now possible to complete the Equation (7) and determine the costs incurred for choosing this option. Inserting all probabilities creates the Equation (27) that results in the total cost for that standby option:

$$
\begin{aligned}
C_{T}= & C_{m 1}+C_{m 2}+C_{a 1} *\left(P_{A a}+P_{A r}\right)+C_{a 2} *\left(P_{a A}+P_{r A}\right)+C_{r 1} *\left(P_{r A}+P_{r r}\right)+ \\
& +C_{r 1} *\left(P_{A r}+P_{r r}\right)+C_{s 1} * P_{r A}+C_{s 2} * P_{A r}+C_{o 1} * P_{a A}+C_{o 2} * P_{A a}+C_{p} * P_{r r}
\end{aligned} .
$$

To enable a meaningful cost comparison with the option in 3.1 the probabilities for $P_{r r}$ have to be identical between the two options otherwise the reliability of service for both options would not be identical and have to be considered as well.

\section{EXAMPLE APPLICATION}

To show the quantitative implications of the approach the following example problem is constructed. A product-service system provider is developing a new machine to deliver its service to their customers. Now a decision is required on whether the new concept provides enough benefits over the current generation of the machine, already in use with customers, to continue development. The company currently uses two identical machines are provided at the customer location for delivery of the service, one of the machines is active and the other one is in cold standby to ensure the availability 
required by the customer. The customer finds it acceptable if the service is unavailable in $0.01 \%$ of its time in operation. Therefore the inability to continue the service $P_{p}$ cannot be greater than $0.01 \%$ otherwise the provided service would no longer be acceptable for the customer. This means there are two possible options have been narrowed down to two final options. Option 1 which would only require a single machine to meet the customer requirements for availability and option 2 with two machines, in which one is held in cold standby as before. All relevant costs have been determined and have been calculated for a total time of operation of 9000 hours. Failure and repair rates have been determined through simulation and testing of prototypes and both achieve the required service availability. The resulting data is compiled in Table 3. Since the both options full fill the technical requirements, an economic comparison is made to determine whether the continuation of development is a good decision.

Table 3. Information about the machines for both options

\begin{tabular}{|l|l|l|l|c|c|c|c|c|}
\hline & \multicolumn{1}{|c|}{$\lambda$} & $\mu$ & \multicolumn{1}{c}{$C_{m}$} & $C_{a}$ & $C_{r}$ & \multicolumn{1}{c}{$C_{s}$} & \multicolumn{1}{c|}{$C_{o}$} & $C_{p}$ \\
\hline Option 1 & 0.00004 & 0.4 & $100,000 €$ & $10,000 €$ & $60,000 €$ & N/A & N/A & $100,000 €$ \\
\hline Option 2 & 0.0053 & 0.35 & $40,000 €$ & $12,000 €$ & $20,000 €$ & $18,000 €$ & $10,000 €$ & $100,000 €$ \\
\hline
\end{tabular}

Looking at the data compiled in this table an obvious financial advantage for either option is not easily visible, because the increase in price cannot be directly related to the lowered failure rate. Therefore the approach described in section 3 is used to calculate the total costs for both options. For the single machine option Equation (13) can be used. Resulting in the costs of option one $C_{T 1}$ :

$$
\begin{aligned}
C_{T 1} & =100,000 €+10,000 € * \frac{0.4}{0.00004+0.4}+60,000 € * \frac{0.00004}{0.00004+0.4}+100,000 € * \frac{0.00004}{0.00004+0.4} \\
& =110,014.99 € .
\end{aligned}
$$

To calculate the costs for option two the Equation (27) is used with the simplified Equations (22)-(26) resulting in the total cost for option two during the defined usage period:

$$
\begin{aligned}
C_{T 2}= & 2 * 40,000 €+2 * 12,000 € *(0.4925+0.0075)+2 * 20,000 € * \\
& *(0.0075+0.0001)+2 * 18.000 € * 0.0075+2 * 10,000 € * 0.4925+ \\
& +100,000 € * 0.0001=102,430.95 € .
\end{aligned}
$$

The comparison of $C_{T 1}$ and $C_{T 2}$ reveals that option two is preferable from a financial standpoint, since it fulfils the customer requirements on availability while incurring lower costs for the same service provided and therefore a better profit margin. Since in this example the availability of the service has to be $99.99 \%$ Equations (28) and (29) show that the driving cost factors are the initial investment for the machines along with the operation and opportunity cost. Changes in those cost would in turn change the preferred option.

\section{CONCLUSION AND OUTLOOK}

During product development multiple different concepts are developed which would fulfil the specified requirements and are technically possible. Choosing between these different options is an important step, because it has to be ensured that the option is chosen which will promise the highest profits for the company. This is especially complicated for result oriented product service system providers since they incur all costs related to providing the sold service. With the approach shown in this paper it is possible to quickly compare two or more options with different failure and repair rates to evaluate their total cost to provide the required service. This enables fast and feasible comparisons between different options while also being able to make small adjustments to the different options to evaluate how certain changes to the product used for service delivery would affect the preferred option.

Further improvement of the approach could be achieved through inclusion of the scrap costs of the products and showing how the system costs would change for more than one standby machine. A consideration of the fact that product-service systems are usually realized using a fleet of identical products should also be taken in consideration in future work. Because this could have a further impact on which option has better cost efficiency for the entire product-service system. Breaking down the product structure would also be worthwhile to identify which subassemblies and components 
have the biggest impact on the life cycle cost. Additionally the implementation of a risk assessment could prove useful since the values used for the calculation are values from early stages of the product development and thus can fluctuate because of uncertainties when obtaining these values. A possible way to realize this could be by incorporating a sensitivity analysis.

\section{REFERENCES}

Chambari, A., Rahmati, S.H.A., Najafi, A.A. and Karimi, A. (2012), “A bi-objective model to optimize reliability and cost of system with a choice of redundancy strategies", Computers \& Industrial Engineering, Vol. 63 No. 1, pp. 109-119.

Chern, M.-S. (1992), "On the computational complexity of reliability redundancy allocation in a series system", Operations Research Letters, Vol. 11 No. 5, pp. 309-315.

Coit, D.W. (2003), "Maximization of System Reliability with a Choice of Redundancy Strategies", IIE Transactions, Vol. 35 No. 6, pp. 535-543.

Ebeling, C.E. (2010), An introduction to reliability and maintainability engineering, 2. ed., Waveland Press, Long Grove Ill.

Eberlin, S. and Hock, B. (2014), Zuverlässigkeit und Verfügbarkeit technischer Systeme, Springer Fachmedien Wiesbaden, Wiesbaden.

Ehrlenspiel, K., Kiewert, A., Hundal, M.S. and Lindemann, U. (2007), Cost-efficient design, Springer, Heidelberg, New York.

Farr, J.V. (2011), Systems life cycle costing: Economics analysis, estimation, and management, Engineering management book series, Taylor \& Francis, Boca Raton.

Goedkoop, M. (1999), Product Service systems, Ecological and Economic Basics.

ISO 15663-1 (2000), "Petroleum and natural gas industries", Life cycle costing, BSI British Standards, London.

Johannknecht, F. (2018), Lebenszyklusorientiertes Kostenmanagement für Produkt-Service Systeme: Dissertation, Berichte aus dem iPeG, 2017, Band 5, PZH Verlag, Garbsen.

Johannknecht, F., Gatzen, M.M., Hahn, D. and Lachmayer, R. (2016a), "Holistic Life Cycle Costing Approach for Different Development Phases of Drilling Tools", in IPTC, 2016/11/12, International Petroleum Technology Conference, p. 11.

Johannknecht, F., Gatzen, M.M. and Lachmayer, R. (2016b), "Life Cycle Cost Model for Considering Fleet Utilization in Early Conceptual Design Phases", Procedia CIRP, Vol. 48, pp. 68-72.

Norris, J.R. (1997), Markov Chains, Cambridge University Press, Cambridge.

Ross, S.M. (2014), Introduction to probability and statistics for engineers and scientists, 5. ed., Elsevier Acad. Press, Amsterdam.

Stewart, W.J. (2009), Probability, Markov Chains, Queues, and Simulation: The Mathematical Basis of Performance Modeling, Princeton University Press, New Jersey.

Tukker, A. (2004), "Eight types of product-service system: eight ways to sustainability? Experiences from SusProNet", Business Strategy and the Environment, Vol. 13 No. 4, pp. 246-260. 